Ann. Abeille, I96r, 4 (4), 36r-367.

\title{
DEUX NOUVEAUX APPAREILS POUR LA PESÉE DES RUCHES
}

\author{
J. FRESNAYE \\ Station expérimentale d'Apiculture, Centre de Recherches agronomiques du Sud-Est, \\ Montfavet (Vaucluse).
}

\section{SOMMAIRE}

Description de deux nouveaux appareils qui facilitent la pesée des ruches :

Un dynamomètre dont la simplicité de namipulation permet la pesée rapide d'un grand nombre de ruches.

Une bascule hydraulique enregistreuse qui permet de pratiquer la pesée permanente d'une ruche.

Ces appareils sont d'une grande utilité pour l'apiculture pratique comme pour l'apiculture expérimentale.

\section{I. - INTRODUC'TION}

la pesée des ruches est essentielle pour la conduite du rucher expérimental comme pour celle du rucher de rapport ; elle permet d'assurer aux Abeilles un bon hivernage, de sélectionner les meilleurs souches et de connaitre l'effet des interventions expérimentales. Une très grande précision n'est pas nécessaire ; une erreur de quelques centaines de grammes sur les $30 \mathrm{~kg}$ à $40 \mathrm{~kg}$ d'une ruche est pratiquement négligeable. Par contre il est indispensable de disposer d'appareils permettant de peser rapidement et facilement un grand nombre de ruches.

\section{II. - MÉTHODES UTILISÉES JUSQU'ICI}

Les appareils utilisés jusqu'à présent étaient d'une précision satisfaisante mais la pesée se faisait avec une telle lenteur ou complication que la plupart des apiculteurs les ont abandonnés. I.es dynamomètres ou pesons nécessitent généralement des systèmes d'accrochages compliqués. Les balances romaines doivent être suspendues et sont munies de curseurs qu'il faut déplacer. Les différents types de bascules, romaines, décimales, automatiques, exigent une mise en place méticuleuse. Les opérations peuvent paraître simples ; mais lorsqu'il faut les répéter cent fois ou davantage dans la même journée elles deviennent impraticables. Pour devenir possible la pesée des ruches doit s'opérer en quelques secondes seulement. 


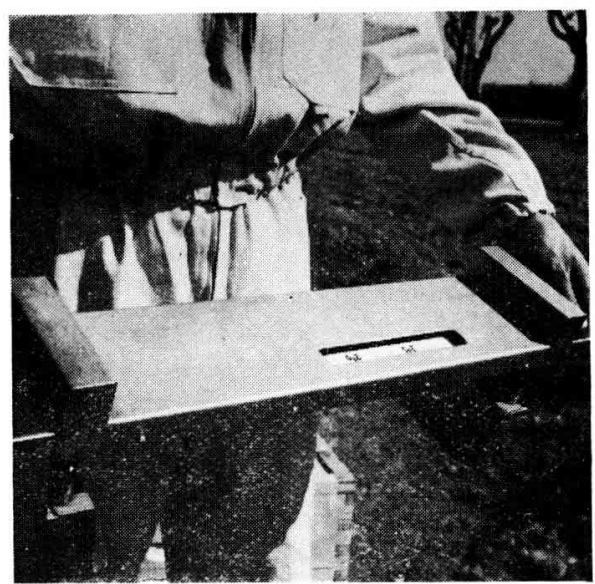

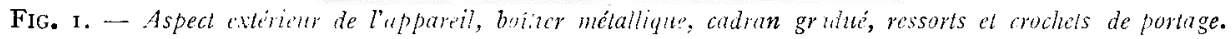

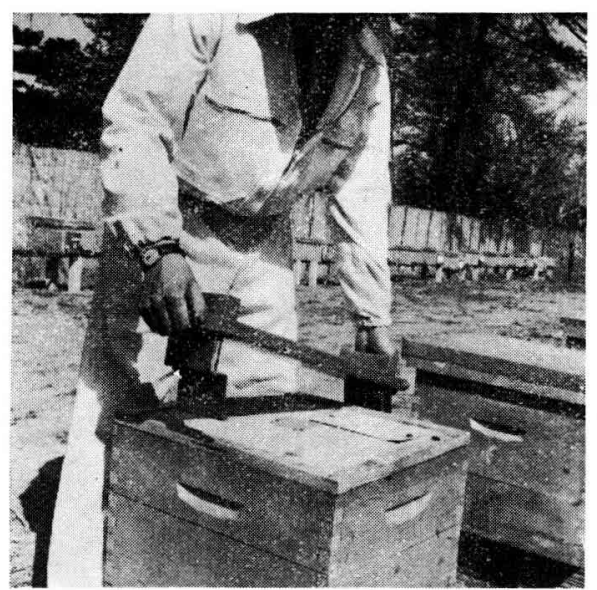

FIG. 2. - Accrochuse de la ruche. Les crochets s'encastrent aulonatiquement dans les entailles de colle-ci.

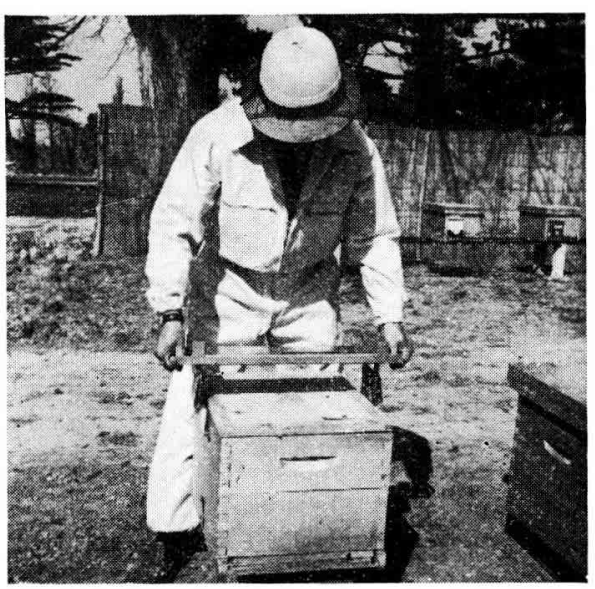

Jiki. 3. --- Pesée ile la ruche. Soulerter la ruche à l'aìde de l'appareil, le pouds est immédiatement lisible sur le cudran. Refoser la nohe sins secousse. 
En général les apiculteurs se contentent de soupeser leurs ruches en dépit des erreurs grossières qui peuvent découler de l'estimation musculaire des poids. L,es bascules enregistreuses pour la pesée permanente des ruches sont très fragiles et ne peuvent être utilisées à l'extérieur. LUTZ (I960) a construit un appareil très simple qui permet d'évaluer approximativement l'augmentation de poids d'une colonie; il peut être utilisé à l'extérieur mais ne comporte pas de système d'enregistrement.

Les différents inconvénients des appareils utilisés jusqu'ici et la nécessité de peser toutes les colonies de notre rucher expérimental m'ont amené à construire un appareil qui permet de peser très rapidement les ruches tout en conservant une précision suffisante. Il est utilisable également dans différentes branches de l'agriculture et de l'industrie, mais il remplit particulièrement bien les conditions requises par l'apiculture moderne.

\section{III. - APPAREIL POUR LA PESÉE RAPIDE DES RUCHES}

Il se classe dans la catégorie des dynamomètres. Sa forme générale est parallélépipédique, il est très peu encombrant et dépasse de peu la largeur d'une ruche. Il est constitué principalement par un chassis métallique muni à ses extrémités de poignées de portage. Quatre ressorts sont reliés d'une part au chassis, d'autre part à deux crochets qui s'adaptent anx entailles de portage de la ruche. Une aiguille solidaire des ressorts se déplace sur un cadran gradué (fig. I). Il suffit de tenir l'appareil par les poignées destinées à cet effet et de le présenter au-dessus dela ruche, les crochets s'encastrent automatiquement dans les entailles dela ruche grâce à la rigidité des ressorts (fig. 2) . Il ne reste plus qu'à soulever, lire le poids, poser la ruche (fig. 3). Toutel'opération ne prend que quelques secondes. Outre cette grande rapidité les principaux avantages de l'appareil sont:

- Encombrement réduit permettant un transport facile.

- Suppression de tous liens, sangles ou autre système de fixation long à installer.

- Aucune installation préalable, pas de socle ou de pied.

- Utilisation indifféremment par une ou deux personnes.

- Aucune perturbation causée à la colonie pesée.

Il est nécessaire que les ruches à peser soient toutes de même type ou tout au moins de même largeur, mais ce point ne se discute đéjà plus en apiculture moderne, où la standardisation du matériel progresse rapidement.

\section{IV. - APPAREII, POUR IA PESÉE PERMANENTE DES RUCHES.}

Fn dehors des pesées périodiques pratiquées sur toutes les ruches d'une exploitation il est parfois utile de peser de façon permanente un certain nombre de colonies. Dans ce domaine comme dans celui de la pesée périodique les moyens mis à la disposition des chercheurs et des apiculteurs sont insuffisants ; ou les bascules ne sont pas 


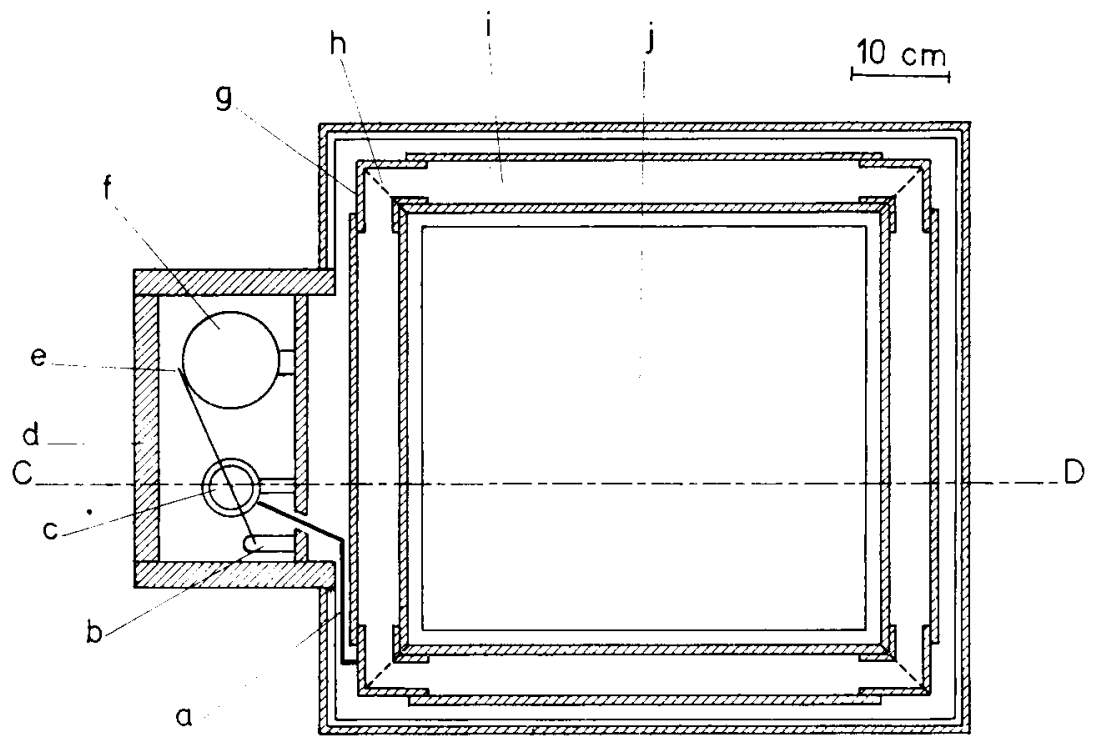

Fig. 4. - Coupe A B.

Coupe vertical: de l'appareil ou les pièces et parties désignées sont :

a) Conduit souple reliant les tubes de caoutchouc au réservoir de liquide. - b) Guides assulant la verticalité du flotteur et de la plume. - - c) Réservoir de liquide à niveau variable en fontion du poids posé sur lat bascule. - d) Porte donnant accìs au mécanisme et permettant l'ćchange des diagrammes. - $e$ ) Plume. i) Tube de caoutchouc. - j) Partie de l'apparcil évidée jer mettant le passage des Abeilles dans le cas d'utilisation entre ruche et hausse.

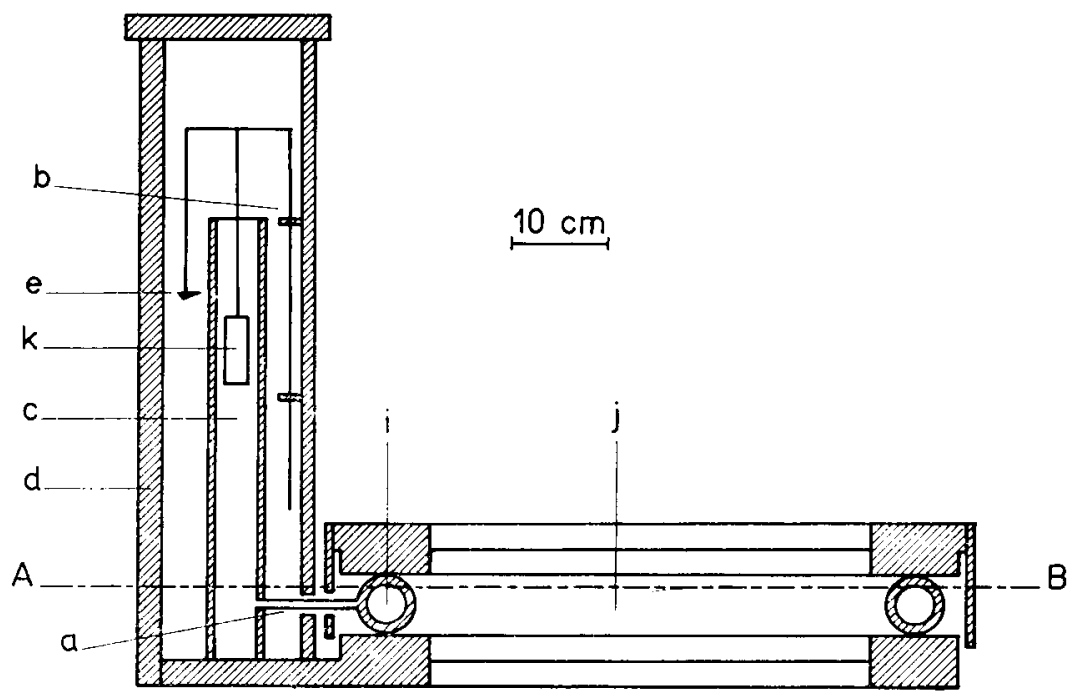

FIG. 5. - Coupe C D.

Coupe en plan de l'apprareil montrant les parties suivantes:

a) Conduit souple relizunt les tubes de caoutchouc au réservoir de liquide. - b) Guides assurant lia verticalité du flotteur et de la plume. - c) Réscrvoir de liquide. - d) Porte donnant accès au mécanisme. e) Plume. - f) Cylindre enregistreur à nouvement d'horlogerie. - g) Tubes métalliques en angle pour assemblage des tubes de caoutchouc. - $k$ ) Cloisons perforées d'un trou de faible diamètre (explication dans le texte). - i) Tubes de caoutchouc. - j) Partie évidée permettant le passage des Abeilles dans le cas de l'utilisation cle apparcil entre ruche et hausse. 


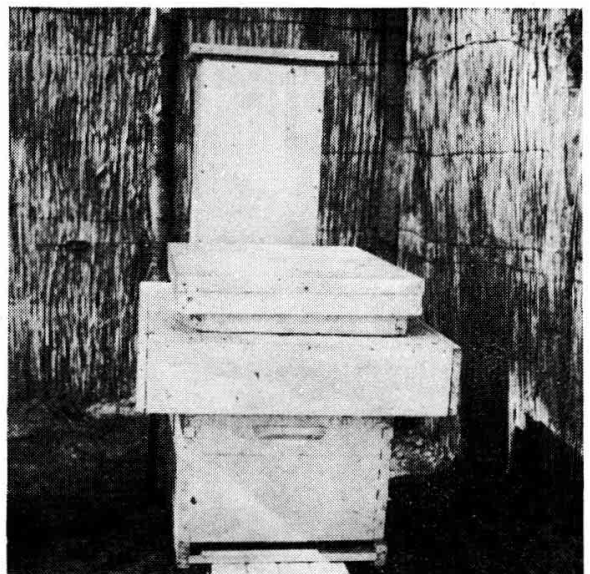

Fín. 6. - Appareil en place pour peser la hausse seule, pentant la récolle dir miel par les abeilles.

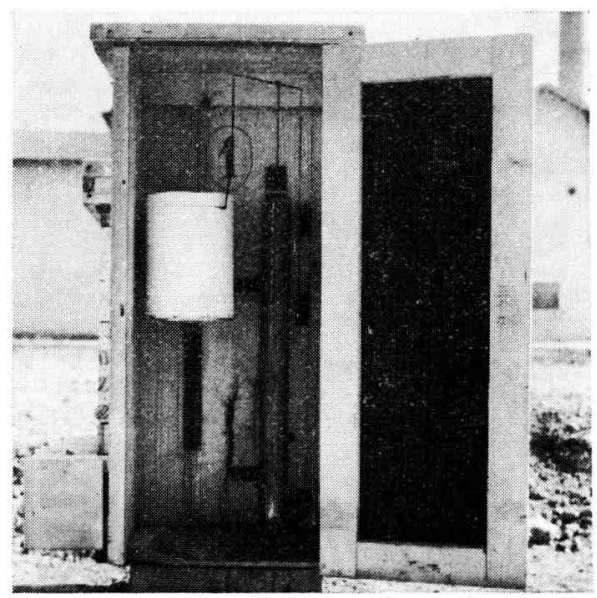

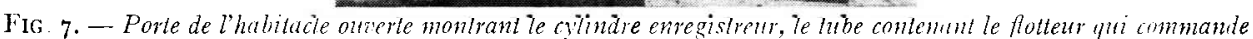

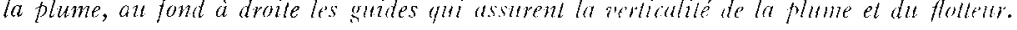

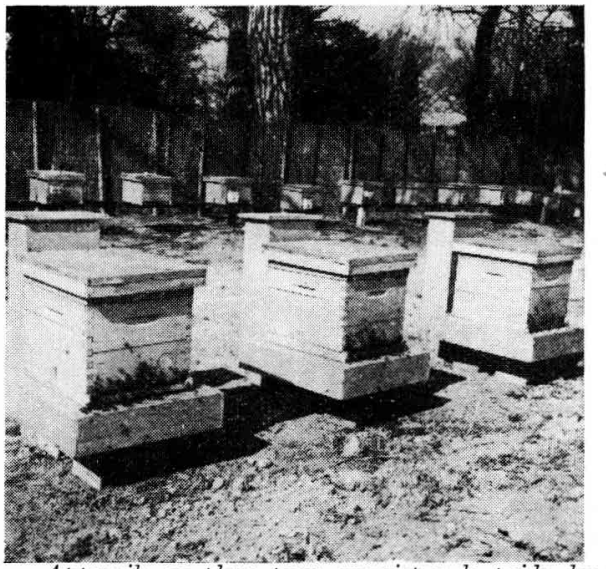

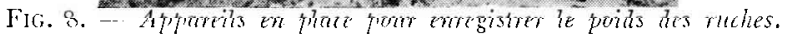


enregistreuses, ou leur mécanisme est trop fragile ce qui interdit leur installation à l'extérieur.

Notre travail expérimental m'a amené à concevoir un appareil très simple, que nous utilisons depuis trois ans et qui, s'il n'est pas parfait, n'a pas les défauts précé-

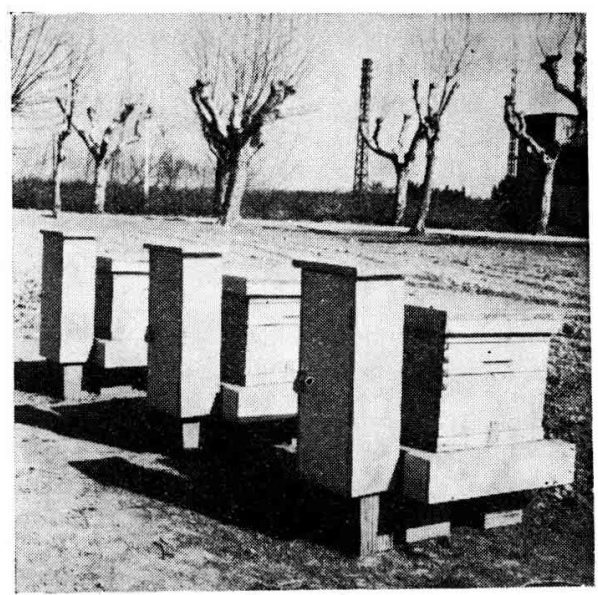

Fıc. 9. -- Vue arrière des appareils en aclizilé.
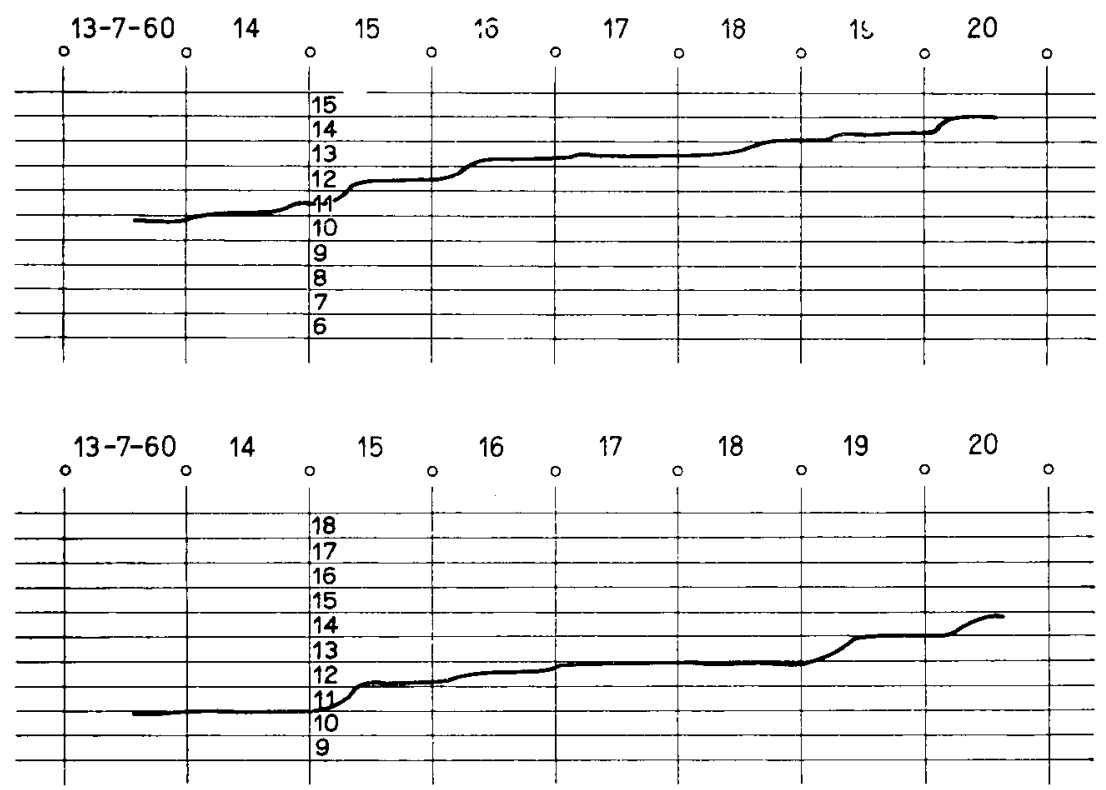

Fig. io. - Fragments des diagrammes de deux ruches équipées de bascules hydrauliques pendant la semaine du $\mathrm{r}_{3}$ au 20 juillet 1960 . Les poids sont indiqué en $\mathrm{kg}$.

demment cités. En outre il est le seul à permettre indifféremment le pesage de la ruche complète ou de la hausse seule pendant la récolte de miel par les Abeilles.

C'est un appareil hydraulique. L'organe principal est un tube de caoutchouc rempli d'eau, sur lequel repose la partie à peser, c'est-à-dire la ruche ou la hausse seule. 
Le liquide déplacé par la pression pénètre dans un tube vertical jusqu'à un niveau qui varie selon l'importance de la charge. Un flotteur se déplace librement dans le tube. Ce flotteur est relié à une plume qui inscrit le poids sur un cylindre enregistreur. Le diamètre des tubes est calculé pour obtenir un déplacement de la plume d'environ I $\mathrm{cm}$ par kilo. On augmente facilement le déplacement de la plume en diminuant le diamètre des tubes. Des cloisons sont placées dans le tube de caoutchouc. Elles sont percées d'un petit orifice pour la circulation du liquide. Elles servent à freiner cette circulation et empêchent le balancement de la hausse au-dessus de la ruche, causé par le ventoules chocs (fig. 4 et 5). Dans lecas oì on ne pèse que la hausse (fig. 6), l'ouverture très large entre le corps de ruche et la hausse permet une facile montée des Abeilles dans celle-ci.

Une toile enduite d'une solution de propolis empêche les Abeilles de pénétrer à l'intérieur de l'appareil et évite la construction de rayons sur les parois.

Un habitacle abrite le cylindre enregistreur et le tube avec son flotteur (fig. 7). L'appareil peut ainsi être exposé aux intempéries sans inconvénient (fig. 8 et 9 ). On peut l'utiliser comme une simple balance en supprimant le cylind re enregistreur et le flotteur et en remplaçant le tube métallique par un tube de verre. Une règle graduée placée derrière ce tube permet la lecture du poids d'après le niveau d'eau.

Au cours de l'année I 960 quatre ruches furent équipées de balances hydrauliques dans les ruchers de notre Station. Nous présentons quelques diagrammes qui montrent avec quelle fidélité ces appareils suivent le déroulement d'une miellée (fig. Io).

\section{CONCLUSION}

La pesée des ruches était jusqu'ici assez compliquée pour que les apiculteurs y renoncent presque toujours. La mise au point d'appareils simples et efficaces permet de remédier à cette carence. Leur emploi permet 1'utilisation du poids en expérimentation apicole. Il assure en outre une parfaite connaissance de l'état des ruches, diminuant ainsi le danger de mortalité.

Reçu pour publication en avril 1961.

\section{SUMMARY}

Description of two new apparatus which makes casy the weighing of hives :

a dynamometer easy to handle for weighing quickly a large number of hives ;

an hydraulic recording scale for permanent weighing of a hive. keeping.

These apparatus are very useful for practical bee-keeping as well as for experimental bee-

\section{RÉFÉRENCE BIBLIOGRAPHIQUE}

Lutz, W., I960. Pour les pesées de ruches. Belg. Apic, то4-го6. 